\title{
Exact traveling wave solutions of modified KdV-Zakharov-Kuznetsov equation and viscous Burgers equation
}

\author{
Md Hamidul Islam, ${ }^{1,2}$ Kamruzzaman Khan ${ }^{3 *}$, M Ali Akbar ${ }^{4}$ and Md Abdus Salam ${ }^{5}$
}

\begin{abstract}
Mathematical modeling of many physical systems leads to nonlinear evolution equations because most physical systems are inherently nonlinear in nature. The investigation of traveling wave solutions of nonlinear partial differential equations (NPDEs) plays a significant role in the study of nonlinear physical phenomena. In this article, we construct the traveling wave solutions of modified KDV-ZK equation and viscous Burgers equation by using an enhanced $(G ' / G)$-expansion method. A number of traveling wave solutions in terms of unknown parameters are obtained. Derived traveling wave solutions exhibit solitary waves when special values are given to its unknown parameters.
\end{abstract}

Keywords: Enhanced (G '/G)-expansion method; Modified KDV-ZK equation; Viscous burgers equation; Traveling wave; Solitary wave

Mathematics subject classification: 35C07; 35C08; 35P99

\section{Background}

Engineers, physicists, and mathematicians have always shown their incessant interest in studying nonlinear problems related to numerous scientific applications, such as fluid dynamics, high-energy physics, plasma physics, elastic media, optical fibers, biomathematics, chemical kinematics, chemical physics and geochemistry. Many young scientists have also shown their increased interest in last two decades because of plausible development of nonlinear science during this period of time. In order to understand the behavior of a nonlinear phenomenon we need to solve the nonlinear equation/set of equations describing that phenomenon, is often very much challenging. There are so many approaches developed over years to analyze/solve such system of nonlinear equations, most of them are based on some assumptions, and hence approximations. Though perturbation methods, like other nonlinear analysis techniques, have their own limitations, are most useful methods among all these

\footnotetext{
* Correspondence: k.khanru@gmail.com

${ }^{3}$ Department of Mathematics, Pabna University of Science and Technology,

Pabna 6600, Bangladesh

Full list of author information is available at the end of the article
}

approaches so far. Using perturbation method, to achieve the ideal results an appropriate choice of small parameter has to be made efficiently, otherwise, a fatal error in results may occur. The perturbation methods are not even applicable to many nonlinear equations because of not having small parameter, which is the principal assumption that has to be met by an equation in order to apply perturbation method. Furthermore, the approximate solutions obtained using perturbation methods are valid only for the small values of the parameters (Ghorbani and Saberi-Nadjafi 2007; Mohiud-Din 2007; Mohyud-Din and Noor, 2009). The investigation of exact traveling wave solutions to these nonlinear equations (NPDEs) have also been observed as a field of great interest to many mathematicians and physicists because of its significant role in understanding the behavior of nonlinear physical phenomena. As a result, numerous techniques of obtaining traveling wave solutions have been developed over last three decades, such as, the Hirota's bilinear transformation method (Hirota 1973, 1981), the modified simple equation method (Jawad et al. 2010; Khan and Akbar 2013a; Ahmed et al. 2013; Zayed and Hoda 2013; Zayed and Arnous 2012), the tanh-function method (Wazwaz 2005; Parkes and Duffy 1996), the Exp-function method (He and Wu 2006; Akbar and Ali 2011b; Bekir and Boz 2008; Xu et al. 2009), the Jacobi elliptic function method (Ali 2011), the 
(G '/G) -expansion method (Akbar et al. 2012a, 2012b; Akbar and Ali 2011a; Wang et al. 2008; Shehata 2010; Koll and Tabi 2011; Naher et al. 2013; Zayed 2009, 2010; Aslan 2010; Bekir and Aksoy 2012), the homotopy perturbation method (Mohiud-Din 2007; Mohyud-Din and Noor 2009), transformed rational function method (Ma and Jyh 2009; Ma and Fuchssteiner 1996), multiple exp-function method (Ma et al. 2010; Ma and Zhu 2012), generalize Hirota bilinear method (Ma 2013), enhanced $\left(G^{\prime} / G\right)$-expansion method (Khan and Akbar 2013b), The Sine-Cosine method (Bibi and Mohyud-Din 2013), the first integral method (Tascan and Bekir 2010; Feng 2002), the ansatz method ( $\mathrm{Hu} 2001 \mathrm{a}, 2001 \mathrm{~b})$ and many others.

The present article is devoted to construct the exact solutions for modified KDV-ZK equation and viscous Burgers equation using a relatively new technique, named, enhanced $\left(G^{\prime} / G\right)$-Expansion method. The rest of the paper is organized as follows. Details explanation of enhanced $\left(G^{\prime} / G\right)$-expansion method has been presented in the next section. The obtained solutions of modified KDV-ZK equation and viscous Burgers equation using this method are presented in Section 3. In Section 4, we have presented some graphs of obtained family of solutions for some particular values of the unknown parameters and the final conclusions are shown in Section 5.

\section{Enhanced ( $G$ ' $/ G$ ) -expansion method}

In this section we describe in details the enhanced $\left(G^{\prime} / G\right)$-expansion method for finding traveling wave solutions of nonlinear evolution equations. Any nonlinear evolution equation in two independent variables $x$ and $t$ can be expressed in following form:

$$
R\left(u, u_{t}, u_{x}, u_{t t}, u_{x x}, u_{x t}, \ldots \ldots \ldots\right)=0
$$

where $u(\xi)=u(x, t)$ is an unknown function, $R$ is a polynomial of $u(x, t)$ and its partial derivatives in which the highest order derivatives and nonlinear terms are involved. The following steps are involved in finding the solution of nonlinear Equation (2.1) using this method (Khan and Akbar 2013b):

Step 1: The given PDE (2.1) can be transformed into ODE using the transformation $\xi=x \pm W t$, where $W$ is the speed of traveling wave such that $W \in R-\{0\}$.

The traveling wave transformation permits us to reduce Eq. (2.1) to the following ODE:

$$
R\left(u, u^{\prime}, u^{\prime \prime}, \ldots \ldots \ldots\right)=0
$$

where $R$ is a polynomial in $u(\xi)$ and its derivatives, where $u^{\prime}(\xi)=\frac{d u}{d \xi}, u^{\prime \prime}(\xi)=\frac{d^{2} u}{d \xi^{2}}$, and so on.
Step 2: We now suppose that Eq. (2.2) has a general solution of the form

$$
u(\xi)=\sum_{i=-n}^{n}\left(\frac{a_{i}\left(G^{\prime} / G\right)^{i}}{\left(1+\lambda\left(G^{\prime} / G\right)\right)^{i}}+b_{i}\left(G^{\prime} / G\right)^{i-1} \sqrt{\sigma\left(1+\frac{\left(G^{\prime} / G\right)^{2}}{\mu}\right)}\right.
$$

subject to the condition that $G=G(\xi)$ satisfy the equation

$$
G^{\prime \prime}+\lambda G=0
$$

where $a_{i}, b_{i}(-n \leq i \leq n ; n \in N)$ and $\lambda$ are constants to be determined, provided that $\sigma= \pm 1$ and $\mu \neq 0$.

Step 3: The positive integer $n$ can be determined by balancing the highest order derivatives to the highest order nonlinear terms appear in Eq. (2.1) or in Eq. (2.2). More precisely, we define the degree of $u(\xi)$ as $D(u(\xi))=$ $n$ which gives rise to the degree of other expression as follows:

$$
D\left(\frac{d^{q} u}{d \xi^{q}}\right)=n+q, D\left(u^{p}\left(\frac{d^{q} u}{d \xi^{q}}\right)^{s}\right)=n p+s(n+q)
$$

Step 4: We substitute Eq. (2.3) into Eq. (2.2) and use Eq. (2.4). We then collect all the coefficients of $\left(G^{\prime} / G\right)^{j}$ and $\left(G^{\prime} / G\right)^{j} \sqrt{\sigma\left(1+\frac{\left(G^{\prime} / G\right)^{2}}{\mu}\right)}$ together. Since Eq. (2.3) is a solution of Eq. (2.2), we can set each of the coefficient equal to zero which leads to a system of algebraic equations in terms of $a_{i}, b_{i}(-n \leq i \leq n ; n \in N), \lambda$, and $W$. One can solves easily these system equations using Maple.

Step 5: For $\mu<0$ general solution of Eq. (2.4) gives

$$
\frac{G^{\prime}}{G}=\sqrt{-\mu} \tanh (A+\sqrt{-\mu} \xi)
$$

and

$$
\frac{G^{\prime}}{G}=\sqrt{-\mu} \operatorname{coth}(A+\sqrt{-\mu} \xi)
$$

And for $\mu>0$, we get

$$
\frac{G^{\prime}}{G}=\sqrt{\mu} \tan (A-\sqrt{\mu} \xi)
$$

and

$$
\frac{G^{\prime}}{G}=\sqrt{\mu} \cot (A+\sqrt{\mu} \xi)
$$

where $A$ is an arbitrary constant. Finally, we can construct a number of families of traveling wave solutions of Eq. (2.1) by substituting the values of $a_{i}, b_{i}(-n \leq i \leq n ; n \in$ $N), \lambda$, and $W$ (obtained in Step 3) and using Eqs. (2.6) (2.9) into Eq. (2.3). 


\section{Applications of enhanced ( $G$ '/G) -expansion method to modified KDV-ZK equation and viscous Burgers equation Modified KDV-ZK equation}

In this current sub-section, we apply enhanced $\left(G^{\prime} / G\right)$ expansion method to solve the modified KDV-ZK equation of the form,

$$
u_{t}+d u^{2} u_{x}+u_{x x x}+u_{x y y}+u_{x z z}=0
$$

where $\mathrm{d}$ is a nonzero constant.

The traveling wave transformation equation $u(\xi)=u$ $(x, t), \quad \xi=x+y+z-W t$ transform Eq. (3.1) into the following ordinary differential equation:

$$
-W u^{\prime}+d u^{2} u^{\prime}+u^{\prime \prime \prime}+u^{\prime \prime \prime}+u^{\prime \prime \prime}=0
$$

Integrating Eq. (3.2) with respect to $\xi$, we obtain

$$
3 u^{\prime \prime}-W u+\frac{1}{3} d u^{3}+R=0
$$

where $R$ is a constant of integration. Following the process as described in step 3 (Section 2), balance between the highest-ordered derivative term $u$ " and nonlinear term $u^{3}$ of Eq. (3.3) provides $n=1$.

For $n=1 \mathrm{Eq}$. (2.3) takes the following form:

$$
\begin{aligned}
u(\xi)= & a_{0}+\frac{a_{1}\left(G^{\prime} / G\right)}{1+\lambda\left(G^{\prime} / G\right)}+\frac{a_{-1}\left(1+\lambda\left(G^{\prime} / G\right)\right)}{\left(G^{\prime} / G\right)} \\
& +b_{0}\left(G^{\prime} / G\right)^{-1} \sqrt{\sigma\left(1+\frac{\left(G^{\prime} / G\right)^{2}}{\mu}\right)} \\
& +b_{1} \sqrt{\sigma\left(1+\frac{\left(G^{\prime} / G\right)^{2}}{\mu}\right)} \\
& +b_{-1}\left(G^{\prime} / G\right)^{-2} \sqrt{\sigma\left(1+\frac{\left(G^{\prime} / G\right)^{2}}{\mu}\right)}
\end{aligned}
$$

where $G=G(\xi)$ satisfies Eq. (2.4). Substituting Eq. (3.4) into Eq. (3.3) and using Eq. (2.4), we get a polynomial in $\left(G^{\prime} / G\right)^{j}$ and $\left(G^{\prime} / G\right)^{j} \sqrt{\sigma\left(1+\frac{\left(G^{\prime} / G\right)^{2}}{\mu}\right)}$. Setting the coefficient of $\left(G^{\prime} / G\right)^{j}$ and $\left(G^{\prime} / G\right)^{j} \sqrt{\sigma\left(1+\frac{\left(G^{\prime} / G\right)^{2}}{\mu}\right)}$ equal to zero, we obtain a system containing a large number of algebraic equations in terms of unknown coefficients. We have solved this system of equations using Maple 13 and obtained the following set of solutions:

Set 1: $R=0, W=-12 \mu, \lambda=0, a_{-1}=\mp \frac{16}{\sqrt{2 d}}, a_{0}=0$, $a_{1}=\mp \frac{16}{\sqrt{2 d}}, \quad b_{-1}=0, \quad b_{0}=0, \quad b_{1}=0$.

Set 2: $R=0, \quad W=6 \mu, \lambda=0, a_{-1}=0, a_{0}=0$, $a_{1}=\mp \frac{16}{\sqrt{2 d}}, b_{-1}=0, b_{0}=0, b_{1}=0$.

Set 3: $R=0, \quad W=\frac{3}{2} \mu, \lambda=0, a_{-1}=0, a_{0}=0$, $a_{1}=\mp \frac{I 3}{\sqrt{2 d}}, b_{-1}=0, b_{0}=0, b_{1}= \pm \frac{I 3}{2} \sqrt{\frac{2 \mu}{\sigma d}}$.
Set 4: $R=0, \quad W=6 \mu, \lambda=\mp \frac{I a_{0}}{6} \sqrt{\frac{2 d}{\mu}}, a_{-1}=\mp \frac{I 6}{\sqrt{2 d}}$, $a_{0}=a_{0}, a_{1}=0, b_{-1}=0, b_{0}=0, b_{1}=0$.

Set 5: $R=0, W=-3 \mu, \lambda=\lambda, a_{-1}=0, a_{0}=0$, $a_{1}=0, \quad b_{-1}=0, \quad b_{0}=0, \quad b_{1}= \pm I 3 \sqrt{\frac{2 \mu}{\sigma d}}$.

Set 6: $R=0, W=-3 \mu, \lambda=\lambda, a_{-1}=0, a_{0}=0$, $a_{1}=0, \quad b_{-1}=0, \quad b_{0}=\mp \frac{I 6 \mu}{\sqrt{2 \sigma d}}, b_{1}=0$.

Corresponding to each set of solution we get two different families of traveling wave solutions of Eq. (3.1) according as $\mu<0$ and $\mu>0$. In doing so, we have obtained twelve families of traveling wave solutions while each family comprises of two solutions.

First we represent the families of hyperbolic solutions corresponding to $\mu<0$ :

Family 1: $\quad u_{1}(\xi)= \pm 3 \sqrt{\frac{2 \mu}{d}}\left(\tanh (A+\sqrt{-\mu} \xi)-\frac{1}{\mu} \operatorname{coth}(A+\sqrt{-\mu} \xi)\right)$, $u_{2}(\xi)= \pm 3 \sqrt{\frac{2 \mu}{d}}\left(\operatorname{coth}(A+\sqrt{-\mu} \xi)-\frac{1}{\mu} \tanh (A+\sqrt{-\mu} \xi)\right)$,

where $\xi=x+y+z+12 \mu t$.

Family 2: $\quad u_{3}(\xi)= \pm 3 \sqrt{\frac{2 \mu}{d}} \tanh (A+\sqrt{-\mu} \xi)$,

where $\xi=x+y+z-6 \mu t$

$$
u_{4}(\xi)= \pm 3 \sqrt{\frac{2 \mu}{d}} \operatorname{coth}(A+\sqrt{-\mu} \xi)
$$

Family 3: $u_{5}(\xi)= \pm 3 \sqrt{\frac{\mu}{2 d}}(\tanh (A+\sqrt{-\mu} \xi)+I \operatorname{sech}(A+\sqrt{-\mu} \xi))$,

$$
u_{6}(\xi)=\mp 3 \sqrt{\frac{\mu}{2 d}}(I \operatorname{coth}(A+\sqrt{-\mu} \xi)+\operatorname{cosech}(A+\sqrt{-\mu} \xi)),
$$

where $\xi=x+y+z-\frac{3}{2} \mu t$.

Family 4: $u_{7}(\xi)=2 a_{0} \mp 3 \sqrt{\frac{2 \mu}{d}} \operatorname{coth}(A+\sqrt{-\mu} \xi)$,

$$
u_{8}(\xi)=2 a_{0} \mp 3 \sqrt{\frac{2 \mu}{d}} \tanh (A+\sqrt{-\mu} \xi),
$$

where $\xi=x+y+z-6 \mu t$.

Family 5: $u_{9}(\xi)=\mp I 3 \sqrt{\frac{2}{\mu d}} \operatorname{cosech}(A+\sqrt{-\mu} \xi) \operatorname{coth}(A+\sqrt{-\mu} \xi)$,

$$
u_{10}(\xi)=\mp 3 \sqrt{\frac{2}{\mu d}} \operatorname{sech}(A+\sqrt{-\mu} \xi) \tanh (A+\sqrt{-\mu} \xi),
$$

where $\xi=x+y+z+3 \mu t$.

Family 6: $u_{11}(\xi)=\mp 3 \sqrt{\frac{2 \mu}{d}} \operatorname{cosech}(A+\sqrt{-\mu} \xi)$,

$$
u_{12}(\xi)=\mp 3 I \sqrt{\frac{2 \mu}{d}} \operatorname{sech}(A+\sqrt{-\mu} \xi),
$$

where $\xi=x+y+z+3 \mu t$.

And, the families of plane periodic solutions corresponding to $\mu>0$ are:

Family 7: $u_{13}(\xi)=\mp I 3 \sqrt{\frac{\mu}{2 d}}\left(\tan (A-\sqrt{\mu} \xi)+\frac{1}{\mu} \cot (A-\sqrt{\mu} \xi)\right)$,

$$
u_{14}(\xi)=\mp I 3 \sqrt{\frac{\mu}{2 d}}\left(\cot (A+\sqrt{\mu} \xi)+\frac{1}{\mu} \tan (A+\sqrt{\mu} \xi)\right),
$$

where $\xi=x+y+z+12 \mu t$.

Family 8: $u_{15}(\xi)=\mp I 3 \sqrt{\frac{2 \mu}{d}} \tan (A-\sqrt{\mu} \xi)$,

$$
u_{16}(\xi)=\mp I 3 \sqrt{\frac{2 \mu}{d}} \cot (A+\sqrt{\mu} \xi),
$$

where $\xi=x+y+z-6 \mu t$. 
Family 9: $u_{17}(\xi)=\mp I 3 \sqrt{\frac{\mu}{2 d}}(\tan (A-\sqrt{\mu} \xi)+\sec (A-\sqrt{\mu} \xi))$,

$$
u_{18}(\xi)=\mp 3 \sqrt{\frac{\mu}{2 d}}(\cot (A+\sqrt{\mu} \xi)+\operatorname{cosec}(A+\sqrt{\mu} \xi)),
$$

where $\xi=x+y+z-\frac{3}{2} \mu t$.

Family 10: $u_{19}(\xi)=2 a_{0} \mp I 3 \sqrt{\frac{2 \mu}{d}} \cot (A-\sqrt{\mu} \xi)$,

$$
u_{20}(\xi)=2 a_{0} \mp I 3 \sqrt{\frac{2 \mu}{d}} \tan (A+\sqrt{\mu} \xi),
$$

where $\xi=x+y+z-6 \mu t$.

Family 11: $u_{21}(\xi)= \pm I 3 \sqrt{\frac{2}{\mu d}} \operatorname{cosec}(A-\sqrt{\mu} \xi) \cot (A-\sqrt{\mu} \xi)$,

$$
u_{22}(\xi)= \pm I 3 \sqrt{\frac{2}{\mu d}} \sec (A+\sqrt{\mu} \xi) \tan (A+\sqrt{\mu} \xi),
$$

where $\xi=x+y+z+3 \mu t$.

Family 12: $u_{23}(\xi)=\mp I 3 \sqrt{\frac{2 \mu}{d}} \operatorname{cosec}(A-\sqrt{\mu} \xi)$,

$$
u_{24}(\xi)=\mp I 3 \sqrt{\frac{2 \mu}{d}} \sec (A+\sqrt{\mu} \xi),
$$

where $\xi=x+y+z+3 \mu t$.
Remark 1: All the obtained solutions have been checked with Maple by putting them back into the original equations and found correct.

\section{Viscous Burgers equation}

In this sub-section, we will apply enhanced $\left(G^{\prime} / G\right)$ expansion method to solve the viscous Burgers equation of the form,

$$
u_{t}+u u_{x}-v u_{x x}=0
$$

where $v$ is the viscosity coefficient. Burgers equation is a model for nonlinear wave propagation, especially in fluid mechanics. It occurs in various areas of applied mathematics, such as modeling of gas dynamics and traffic flow.

The traveling wave transformation equation $u(\xi)=u$ $(x, t), \xi=x-W t$ transform Eq. (3.5) into the following ordinary differential equation:

$$
-W u^{\prime}+u u^{\prime}-v u^{\prime \prime}=0
$$

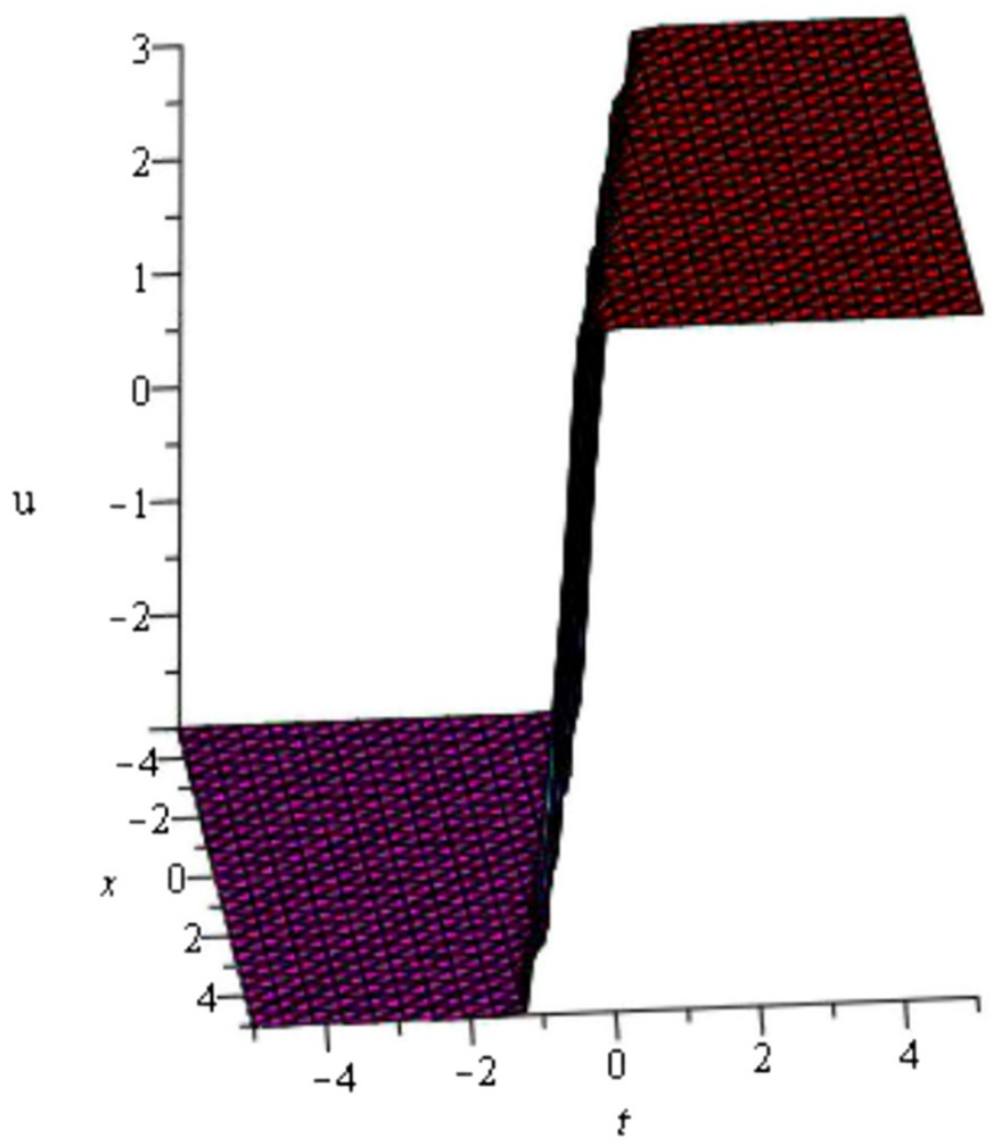

Figure 1 Kink wave profile of mKDV-ZK equation. 
Integrating Eq. (3.6) with respect to $\xi$ setting constant of integration to zero, we obtain

$$
2 v u^{\prime}+2 W u-u^{2}=0
$$

Following the process as described in step 3 (Section 2), balance between the highest-ordered derivative term $u^{\prime}$ and nonlinear term $u^{2}$ of Eq. (3.7) provides $n=1$.

For $n=1 \mathrm{Eq}$. (2.3) takes the following form:

$$
\begin{aligned}
u(\xi)= & a_{0}+\frac{a_{1}\left(G^{\prime} / G\right)}{1+\lambda\left(G^{\prime} / G\right)}+\frac{a_{-1}\left(1+\lambda\left(G^{\prime} / G\right)\right)}{\left(G^{\prime} / G\right)} \\
& +b_{0}\left(G^{\prime} / G\right)^{-1} \sqrt{\sigma\left(1+\frac{\left(G^{\prime} / G\right)^{2}}{\mu}\right)} \\
& +b_{1} \sqrt{\sigma\left(1+\frac{\left(G^{\prime} / G\right)^{2}}{\mu}\right)} \\
& +b_{-1}\left(G^{\prime} / G\right)^{-2} \sqrt{\sigma\left(1+\frac{\left(G^{\prime} / G\right)^{2}}{\mu}\right)}
\end{aligned}
$$

where $G=G(\xi)$ satisfies Eq.(2.4). Substituting Eq. (3.8) into Eq. (3.7) and using Eq. (2.4), we get a polynomial in $\left(G^{\prime} / G\right)^{j}$ and $\left(G^{\prime} / G\right)^{j} \sqrt{\sigma\left(1+\frac{\left(G^{\prime} / G\right)^{2}}{\mu}\right)}$. Setting the coefficient of $\left(G^{\prime} / G\right)^{j}$ and $\left(G^{\prime} / G\right)^{j} \sqrt{\sigma\left(1+\frac{\left(G^{\prime} / G\right)^{2}}{\mu}\right)}$ equal to zero, we obtain a system containing a large number of algebraic equations in terms of unknown coefficients. We have solved this system of equations using Maple 13 and obtained the following set of solutions:

Set 1: $W= \pm v \sqrt{-\mu}, \lambda=0, a_{-1}=0, a_{0}= \pm v \sqrt{-\mu}$,

$$
a_{1}=-v, b_{-1}=0, b_{0}=0, b_{1}= \pm v \sqrt{\frac{\mu}{\sigma}} \text {. }
$$

Set 2: $W=\mp 2 v \sqrt{-\mu}, \lambda=\frac{a_{0} \pm 2 v \sqrt{-\mu}}{2 v \mu}, a_{-1}=0, a_{0}=a_{0}$,

$$
a_{1}=-\frac{a_{0}\left(a_{0} \pm 4 v \sqrt{-\mu}\right)}{2 v \mu}, b_{-1}=0, \quad b_{0}=0, \quad b_{1}=0 .
$$

Set 3: $W= \pm 2 v \sqrt{-\mu}, \lambda=0, a_{-1}=2 v \mu, a_{0}= \pm 2 v$

$\sqrt{-\mu}, a_{1}=0, b_{-1}=0, b_{0}=0, b_{1}=0$.

Set 4: $W= \pm 4 v \sqrt{-\mu}, \lambda=0, a_{-1}=2 v \mu, a_{0}= \pm 4 v \sqrt{-\mu}$,

$a_{1}=0, b_{-1}=0, b_{0}=0, b_{1}=0$.

Set 5: $W= \pm 2 v \sqrt{-\mu}, \lambda=-\left(\frac{a_{0} \mp 2 v \sqrt{-\mu}}{2 v \mu}\right), a_{-1}=2 v \mu$,

$a_{0}=a_{0}, a_{1}=0, b_{-1}=0, b_{0}=0, b_{1}=0$.

Set 6: $W= \pm v \sqrt{-\mu}, \lambda=-\left(\frac{a_{0} \mp v \sqrt{-\mu}}{v \mu}\right), a_{-1}=v \mu$, $a_{0}=a_{0}, a_{1}=0, b_{-1}=0, b_{0}=\frac{v \mu}{\sqrt{\sigma}}, b_{1}=0$.

According to the parallel course of action the families of hyperbolic solutions corresponding to $\mu<0$ are:

Family 1: $\begin{aligned} & u_{1}(\xi)=v \sqrt{-\mu}(1-\tanh (A+\sqrt{-\mu} \xi) \mp I \operatorname{sech}(A+\sqrt{-\mu} \xi)), \\ & u_{2}(\xi)=v \sqrt{-\mu}(1-\operatorname{coth}(A+\sqrt{-\mu} \xi) \mp \operatorname{csch}(A+\sqrt{-\mu} \xi)),\end{aligned}$ where $\xi=x \mp \nu \sqrt{-\mu} t$.

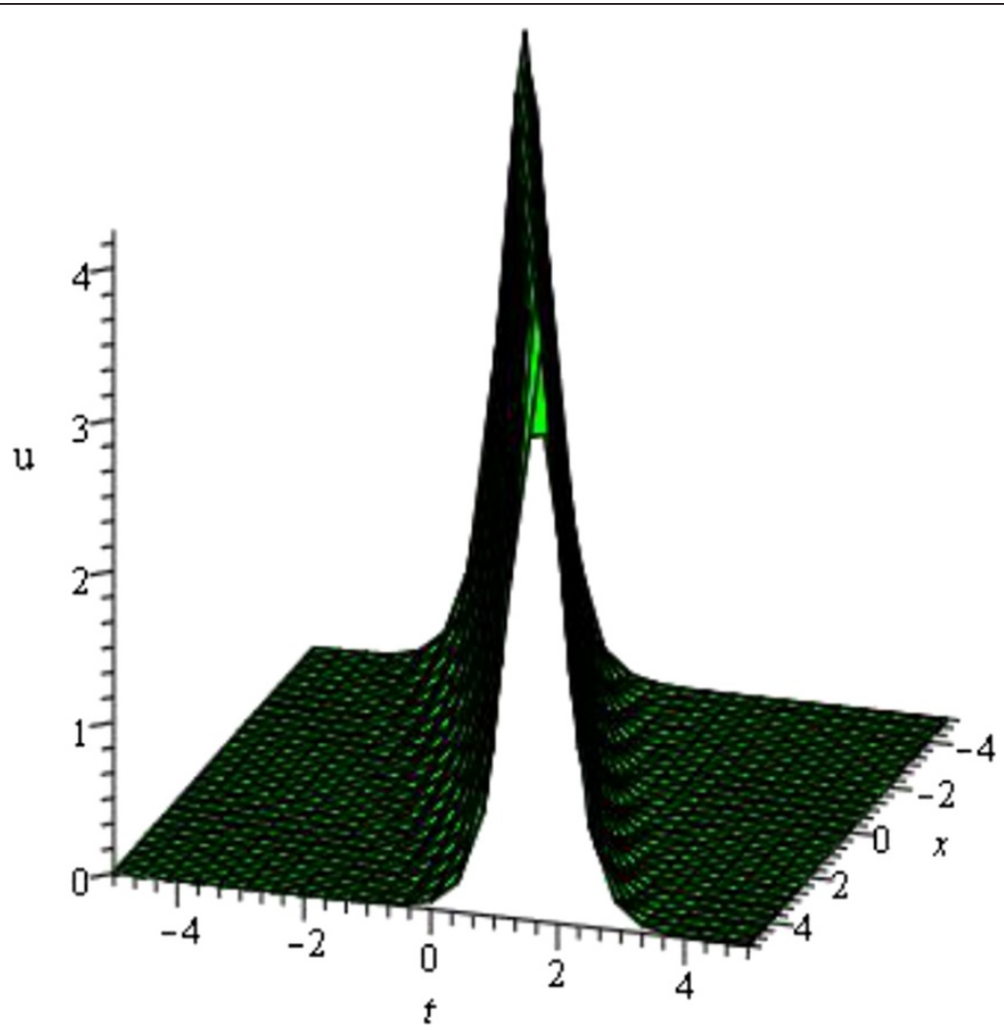

Figure 2 Soliton profile of mKDV-ZK equation. 


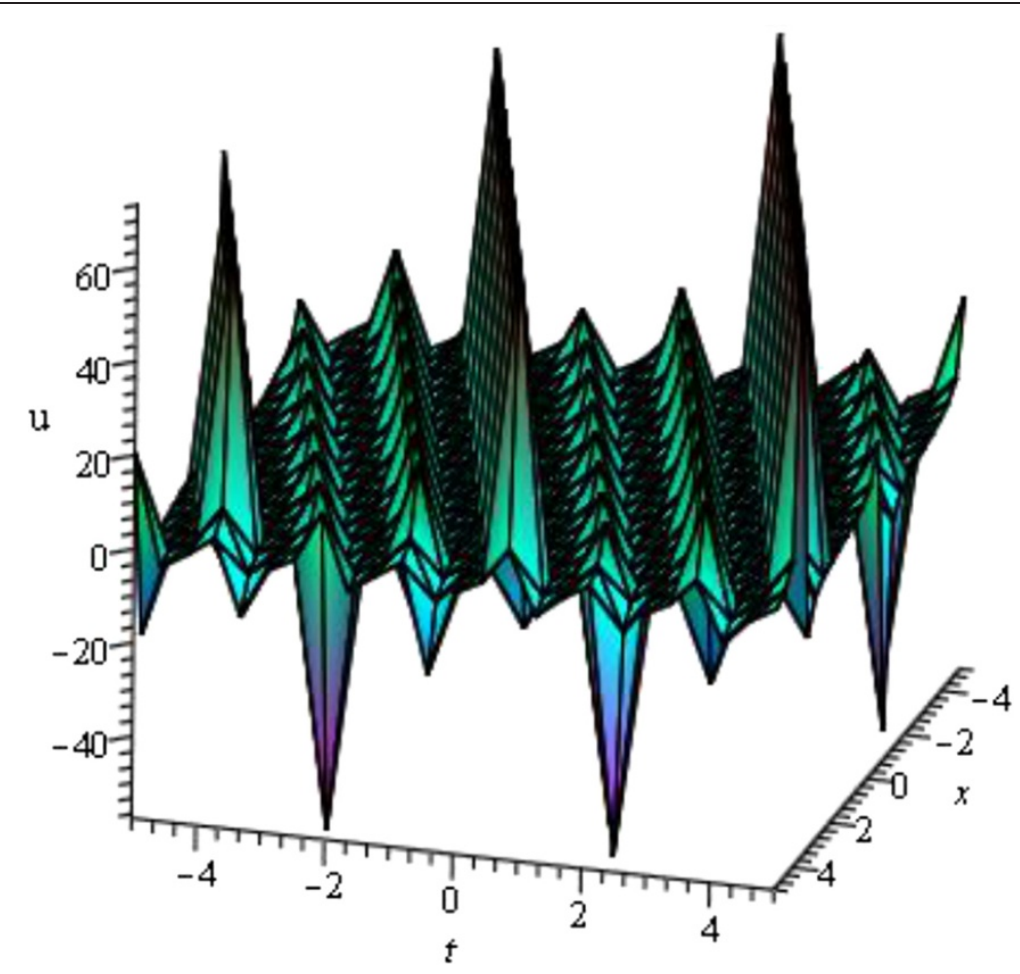

Figure 3 Periodic wave profile of mKDV-ZK equation.

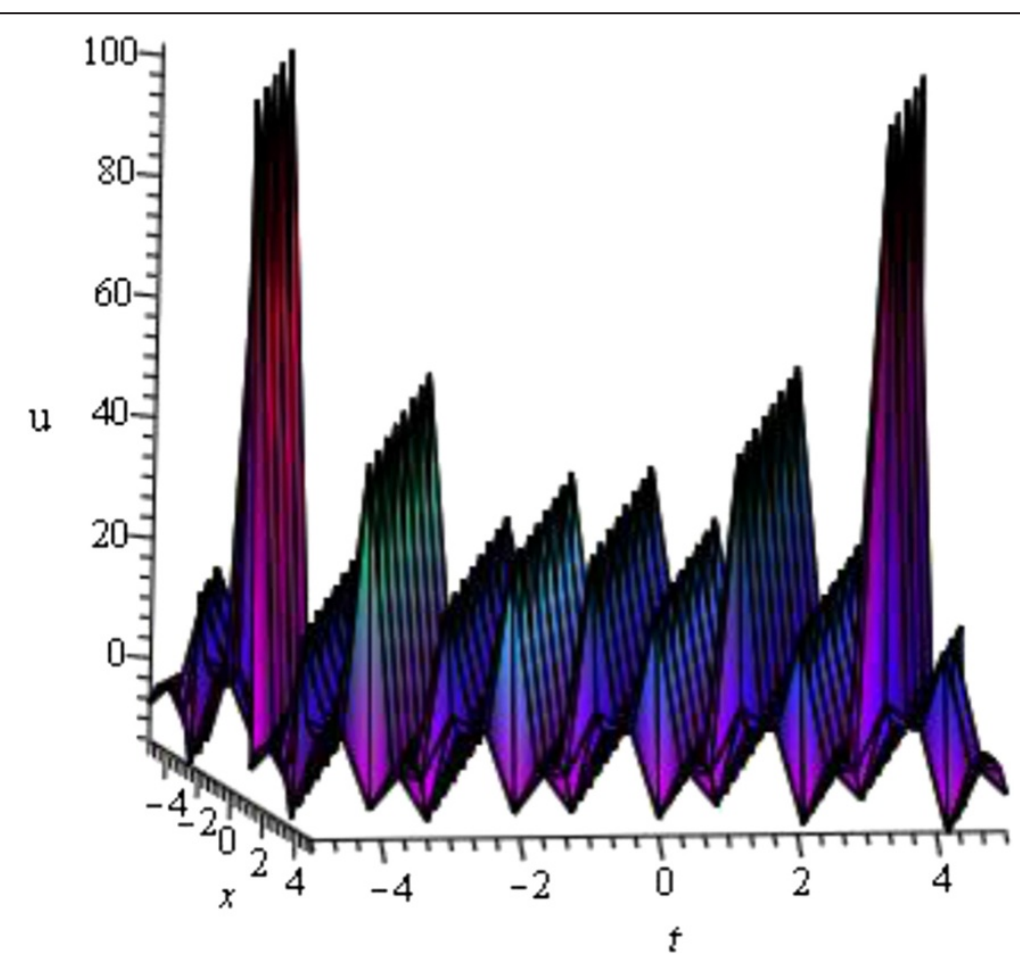

Figure 4 Periodic wave profile of mKDV-ZK equation. 


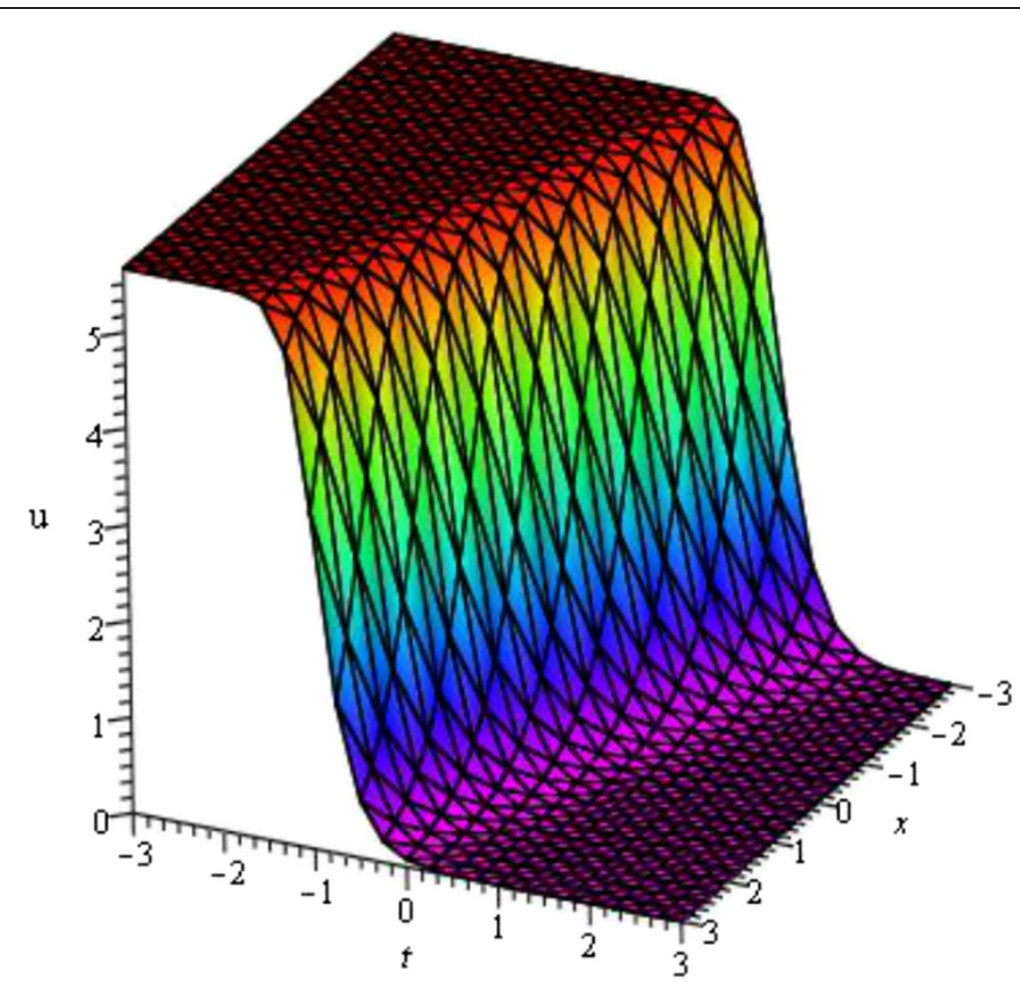

Figure 5 Kink profile of viscous Burgers equation (Shape of $u_{1}(\xi)$ ).

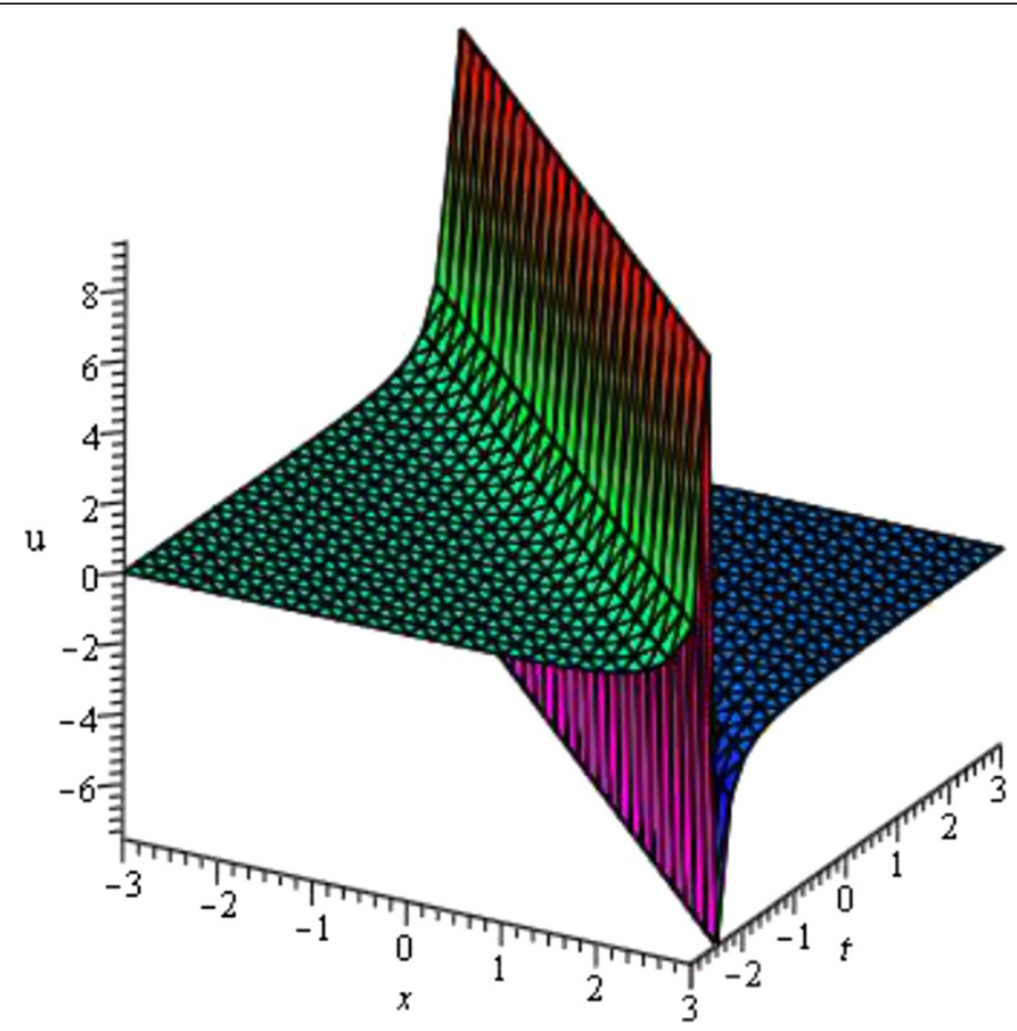

Figure 6 Singular kink profile of viscous Burgers equation (Shape of $u_{3}(\xi)$ ). 
Family 2: $u_{3}(\xi)=2 a_{0} v \mu\left(\frac{1 \pm \tanh (A+\sqrt{-\mu} \xi)}{2 v \mu+\left(a_{0} \sqrt{-\mu} \mp 2 v \mu\right) \tanh (A+\sqrt{-\mu} \xi)}\right)$, $u_{4}(\xi)=2 a_{0} v \mu\left(\frac{1 \pm \operatorname{coth}(A+\sqrt{-\mu} \xi)}{2 v \mu+\left(a_{0} \sqrt{-\mu} \mp 2 v \mu\right) \operatorname{coth}(A+\sqrt{-\mu} \xi)}\right)$,

where $\xi=x \pm 2 v \sqrt{-\mu} t$.

Family 3: $u_{5}(\xi)= \pm 2 v \sqrt{-\mu}(1 \mp \operatorname{coth}(A+\sqrt{-\mu} \xi))$, $u_{6}(\xi)= \pm 2 v \sqrt{-\mu}(1 \mp \tanh (A+\sqrt{-\mu} \xi))$ where $\xi=x \mp 2 v \sqrt{-\mu} t$.

Family 4: $u_{7}(\xi)= \pm 2 v \sqrt{-\mu}(2 \mp \operatorname{coth}(A+\sqrt{-\mu} \xi))$, $u_{8}(\xi)= \pm 2 v \sqrt{-\mu}(2 \mp \tanh (A+\sqrt{-\mu} \xi))$,

where $\xi=x \mp 4 v \sqrt{-\mu} t$.

Family 5: $u_{9}(\xi)= \pm 2 v \sqrt{-\mu}(1 \mp \operatorname{coth}(A+\sqrt{-\mu} \xi))$, $u_{10}(\xi)= \pm 2 v \sqrt{-\mu}(1 \mp \tanh (A+\sqrt{-\mu} \xi))$

where $\xi=x \mp 2 v \sqrt{-\mu} t$.

Family 6: $u_{11}(\xi)=v \sqrt{-\mu}(1-\operatorname{coth}(A+\sqrt{-\mu} \xi) \mp \operatorname{csch}(A+\sqrt{-\mu} \xi))$, $u_{12}(\xi)=v \sqrt{-\mu}(1-\tanh (A+\sqrt{-\mu} \xi) \mp I \operatorname{sech}(A+\sqrt{-\mu} \xi))$

where $\xi=x \mp \nu \sqrt{-\mu} t$.

Similarly, we can write down the other families of exact trigonometric function solutions corresponding to $\mu>0$ which are omitted for convenience.

Remark 2: All the obtained solutions have been checked with Maple by putting them back into the original equations and found correct.

\section{Graphical representation of solitary waves (obtained from above family of traveling waves) Modified KDV-ZK Equation}

We have obtained total twenty four solution profiles of traveling waves in terms of some unknown parameters; subdivided (above) into twelve families according as negative or positive values of $\mu$. All of these solutions are combination of hyperbolic functions or trigonometric functions but Family-4 and Family-10 which are combination of algebraic functions, and hyperbolic functions or trigonometric functions. Solitary waves can be obtained from each traveling wave solution by setting particular values to its unknown parameters. If we put $a_{0}=0$, then Family 4 and Family 10 coincide with Family 2 and Family 8 respectively. In this section, we have presented some graphs of solitary waves constructed by taking suitable values of involved unknown parameters.

- For the values of $\mu=-1, d=-2, A=y=z=0$ within the interval $-5 \leq x, t \leq 5$ Figure 1 is kink wave (only shows the shape of $u_{3}(\xi)$ of mKDV-ZK equation).

- For the values of $\mu=-1, d=1, A=y=z=0$ within the interval $-5 \leq x, t \leq 5$ Figure 2 is soliton wave (only shows the shape of $u_{12}(\xi)$ of mKDV-ZK equation).

- For the values of $\mu=0.50, d=-1, A=3, y=z=0$ within the interval $-5 \leq x, t \leq 5$, Figure 3 is a periodic wave (only shows the shape of $u_{15}(\xi)$ of mKDV-ZK equation).

- For the values of $\mu=1, d=-2, A=y=z=0$ within the interval $-5 \leq x, t \leq 5, u_{24}(\xi)$ (solution of mKDV-ZK equation) shows the shape of periodic wave represented in Figure 4.

\section{Viscous Burgers equation}

Now we will discuss some of the obtained results of viscous Burgers equation and their graphical representations.

For the values of $A=0, \mu=-2, \sigma=1$ and $v=-2$ within the interval $-3 \leq x, t \leq 3$ the solution of viscous Burgers equation $u_{1}(\xi)$ shows Kink wave which is represented in Figure 5 and For the values of $a_{0}=0, A=0$, $\mu=-1, \sigma=-1$ and $v=-0.50$ within the interval $-3 \leq x$, $t \leq 3$ the solution of viscous Burgers equation $u_{3}(\xi)$ shows singular kink wave which is represented in Figure 6. Some graphical representations of viscous Burgers equation are given below:

\section{Conclusions}

In this article, we obtain a large number of exact traveling wave solutions including solitary wave solutions for modified KDV-ZK equation and viscous Burgers equation through enhanced $\left(G^{\prime} / G\right)$ )-expansion method. Using this method, we have found some extra family of solutions, is the best thing about this method. The obtained solutions suggest that enhanced ( $\left.G^{\prime} / G\right)$ )-expansion method can be used as useful mathematical tool for solving nonlinear evolution equations arises in the arena of mathematical physics, engineering sciences and applied mathematics.

\section{Competing interests}

Authors have declared that no competing interests exist.

\section{Authors' contributions}

This work was carried out in collaboration among the authors. All authors have a good contribution to design the study, and to perform the analysis of this research work. All authors read and approved the final manuscript.

\section{Author details}

'Department of Electronics and Telecommunication Engineering, Prime University, Dhaka 1216, Bangladesh. ${ }^{2}$ School of Biomolecular and Physical Sciences, Griffith University, Australia. ${ }^{3}$ Department of Mathematics, Pabna University of Science and Technology, Pabna 6600, Bangladesh. ${ }^{4}$ Department of Applied Mathematics, University of Rajshahi, Rajshahi 6205, Bangladesh.

${ }^{5}$ Department of Mathematics, Mawlana Bhashani Science and Technology University, Tangail 1902, Bangladesh.

Received: 23 July 2013 Accepted: 31 January 2014 Published: 21 February 2014

\section{References}

Ahmed MT, Khan K, Akbar MA (2013) Study of nonlinear evolution equations to construct traveling wave solutions via modified simple equation method. Physical Review \& Research International 3(4):490-503

Akbar MA, Ali NHM (2011a) The alternative (G'/G)-expansion method and its applications to nonlinear partial differential equations. Int J Phys Sci 6(35):7910-7920 
Akbar MA, Ali NHM (2011b) Exp-function method for Duffing equation and new solutions of $(2+1)$ dimensional dispersive long wave equations. Prog Appl Math 1(2):30-42

Akbar MA, Ali NHM, Zayed EME (2012a) Abundant exact traveling wave solutions of the generalized Bretherton equation via $\left(G^{\prime} / G\right)$-expansion method. Commun Theor Phys 57:173-178

Akbar MA, Ali NHM, Mohyud-Din ST (2012b) The alternative ( $\left.G^{\prime} / G\right)$-expansion method with generalized Riccati equation: application to fifth order $(1+1)$ dimensional Caudrey-Dodd-Gibbon equation. Int J Phys Sci 7(5):743-752

Ali AT (2011) New generalized Jacobi elliptic function rational expansion method. J Comput Appl Math 235:4117-4127

Aslan I (2010) Analytic solutions to nonlinear differential-difference equations by means of the extended $\left(\mathrm{G}^{\prime} / \mathrm{G}\right)$ expansion method. J Phys A Math Theor 43:395207(10pp)

Bekir A, Aksoy E (2012) Exact solutions of shallow water wave equations by using the ( $\left.G^{\prime} / G\right)$-expansion method. Waves Random Complex Media 22(3):317-331

Bekir A, Boz A (2008) Exact solutions for nonlinear evolution equations using Exp-function method. Phy Lett A 372:1619-1625

Bibi S, Mohyud-Din ST (2013) Traveling wave solutions of KdVs using sine-cosine method. J Assn Arab Univ Basic Appl Sci. doi: org/10.1016/j.jaubas. 2013.03 .006 (in press)

Feng $Z$ (2002) The first-integral method to study the Burgers-Korteweg-de Vries equation. J Phys A 35(2):343-349

Ghorbani A, Saberi-Nadjafi J (2007) He's Homotopy Perturbation Method for Calculating Adomian Polynomials. Int J Nonlinear Sci Numer Simul 8(2):229-232

He JH, Wu XH (2006) Exp-function method for nonlinear wave equations. Chaos Solitons and Fract 30:700-708

Hirota R (1973) Exact envelope soliton solutions of a nonlinear wave equation. J Math Phy 14:805-810

Hirota R, Satsuma J (1981) Soliton solutions of a coupled KDV equation. Phy Lett A 85:404-408

$\mathrm{Hu} J \mathrm{~L}$ (2001a) A new method for finding exact traveling wave solutions to nonlinear partial differential equations. Phys Lett A 286:175-179

$\mathrm{Hu} J \mathrm{~L}$ (2001b) Explicit solutions to three nonlinear physical models. Phys Lett A 287:81-89

Jawad AJM, Petkovic MD, Biswas A (2010) Modified simple equation method for nonlinear evolution equations. Appl Math Comput 217:869-877

Khan K, Akbar MA (2013a) Exact and solitary wave solutions for the TzitzeicaDodd-Bullough and the modified KdV-Zakharov-Kuznetsov equations using the modified simple equation method. Ain Shams Eng J 4:903-909. doi: org/10.1016/j.asej.2013.01.010

Khan K, Akbar MA (2013b) Traveling wave solutions of nonlinear evolution equations via the enhanced ( $\left.\mathrm{G}^{\prime} / \mathrm{G}\right)$-expansion method. Journal of the Egyptian Mathematical Society. doi.org/10.1016/j.joems.2013.07.009. (In Press)

Koll GR, Tabi CB (2011) Application of the ( $\left.G^{\prime} / G\right)$-expansion method to nonlinear blood flow in large vessels. Phys Scr 83:045803(6pp)

Ma WX (2013) Bilinear equations, Bell polynomials and linear superposition principle. J Phys Conf Ser 411:012021. doi:10.1088/1742-6596/411/1/012021

Ma WX, Fuchssteiner B (1996) Explicit and exact solutions to a KolmgorovPetrovskii-Piskunov equation. Int J of Non-Linear Mechanics 31(3):329-38

Ma WX, Jyh HL (2009) A transformed rational function method and exact solutions to the $(3+1)$-dimensional Jimbo-Miwa equation. Chaos Solitons Fractals 42(3):1356-1363. doi.org/10.1016/j.chaos.2009.03.043

Ma WX, Zhu Z (2012) Solving the (3+1)-dimensional generalized KP and BKP equations by the multiple exp-function algorithm. Appl Math Comput 21811871:79. doi.org/10.1016/j.amc.2012.05.049

Ma WX, Huang T, Zhang Y (2010) A multiple exp-function method for nonlinear differential equations and its application. Phys Scr 82:065003(8pp). doi:10.1088/0031-8949/82/06/065003

Mohiud-Din ST (2007) Homotopy perturbation method for solving fourth-order boundary value problems. Math Prob Engr:1-15. doi:10.1155/2007/98602

Mohyud-Din ST, Noor MA (2009) Homotopy perturbation method for solving partial differential equations. Zeitschrift für Naturforschung. A Journal of Physical Sciences 64a:157-170

Naher H, Abdullah FA, Akbar MA (2013) Generalized and improved (G'/G)expansion method for $(3+1)$-dimensional modified KdV-Zakharov-Kuznetsev equation. PLOSONE 8(5):e64618. doi:10.1371/journal.pone.0064618

Parkes EJ, Duffy BR (1996) An automated tanh-function method for finding solitary wave solutions to non-linear evolution equations. Comput Phys Commun 98:288-300
Shehata AR (2010) The traveling wave solutions of the perturbed nonlinear Schrodinger equation and the cubic-quintic Ginzburg Landau equation using the modified $\left(\mathrm{G}^{\prime} / \mathrm{G}\right)$-expansion method. Appl Math Comput 217:1-10

Tascan F, Bekir A (2010) Applications of the first integral method to nonlinear evolution equations. Chin Phys B 19(8):080201

Wang M, Li X, Zhang J (2008) The ( $\left.\mathrm{G}^{\prime} / \mathrm{G}\right)$-expansion method and travelling wave solutions of nonlinear evolution equations in mathematical physics. Phys Lett A 372:417-23

Wazwaz AM (2005) The tanh-function method: solitons and periodic solutions for the Dodd-Bullough-Mikhailov and the Tzitzeica-Dodd-Bullough equations. Chaos Solitons and Fractals 25(1):55-63

Xu F, Yan W, Chen YL, Li CQ, Zhang YN (2009) Evaluation of two-dimensional ZKMEW equation using the Exp-function method. Comput Math Appl 58:2307-12

Zayed EME (2009) New traveling wave solutions for higher dimensional nonlinear evolution equations using a generalized $\left(G^{\prime} / G\right)$-expansion method. J Phys A Math Theor 42:195202. 13pp)

Zayed EME (2010) Traveling wave solutions for higher dimensional nonlinear evolution equations using the $\left(\mathrm{G}^{\prime} / \mathrm{G}\right)$-expansion method. J Appl Math \& Informatics 28(1-2):383-95

Zayed EME, Arnous AH (2012) Exact solutions of the nonlinear ZK-MEW and the Potential YTSF equations using the modified simple equation method. AIP Conf Proc 1479:2044. doi: 10.1063/1.4756591

Zayed EME, Hoda ISA (2013) Modified simple equation method and its applications for some nonlinear evolution equations in mathematical physics. Int J Comp Appl 67:6 (0975 - 8887)

\section{doi:10.1186/2193-1801-3-105}

Cite this article as: Islam et al.: Exact traveling wave solutions of modified KdV-Zakharov-Kuznetsov equation and viscous Burgers equation. SpringerPlus 2014 3:105.

\section{Submit your manuscript to a SpringerOpen ${ }^{\odot}$ journal and benefit from:}

- Convenient online submission

- Rigorous peer review

- Immediate publication on acceptance

- Open access: articles freely available online

- High visibility within the field

- Retaining the copyright to your article

Submit your next manuscript at $>$ springeropen.com 